ИЗВЕСТИЯ АКАДЕМИИ НАУК ЭСТОНСКОИ ССР, ФИЗИКА * МАТЕМАТИКА PROCEEDINGS OF THE ACADEMY OF SCIENCES OF THE ESTONIAN SSR. PHYSICS * MATHEMATICS

$1986,35,1$

\title{
ВЫЧИСЛЕНИЕ ПОТЕНЦИАЛА ИОНИЗАЦИИ ПО ЧИСЛУ ВЗАИМОДЕЙСТВИЙ МЕЖДУ СТРУКТУРНЫМИ ЕДИНИЦАМИ
}

\section{1. Алкины}

\section{(Представил Я. Эйнасто)}

Расширение уравнения Тафта весьма продуктивно для количественного описания первого потенциала ионизации (ПИ). В случае изучения алкильных соединений дополнительно учитывается влияние числа водородных атомов в двух соседних позициях по отношению к центру ионизации $\left[{ }^{1}\right]$. Пятипараметровое линейное уравнение $\left[{ }^{2}\right]$ описывает большинство известных ПИ разных классов соединений с точностью до 0,2 эВ. Как показал уже В. Ч. Прайс, рост числа алкильных заместителей вызывает неаддитивное уменьшение ПИ $\left[{ }^{3}\right]$, однако в [ $\left.{ }^{4}\right]$ изменение ПИ описано по числу метильных групп в заместителях.

Формальный подход к взаимодействию в рамках принципа полилинейности $\left[{ }^{5}\right]$ и затуханию влияния атомов углерода с удалением от центра ионизации $\left[{ }^{6,7}\right]$ является основой настоящего взгляда относительно роли аддитивных взаимодействий между структурными элементами в изменениях первого ПИ алкильных соединений.

Алкины имеют хорошо локализованный центр ионизации в виде группы $-\mathrm{C} \equiv \mathrm{C}-$ и значительный диапазон изменения ПИ $(2,4$ эВ). Это значит, что ПИ у них исключительно чувствителен к изменениям строения алкильной группы. Все известные значения первого адиабатического ПИ алкинов [4,8,9-11] представлены в табл. 1. Средняя точность всех данных около 0,02 эВ. Данные исследованных методом фотоэлектронной спектроскопии (ФЭС) 36 соединений взяты из $\left[{ }^{8}\right]$ (в табл. 1 выделены). Для этих соединений ПИ измерены с высокой точностью (среднеквадратичная ошибка 0,007 эВ).

Обозначим в алкильном соединении типа $R^{\prime}-Y-R$ позиции атомов согласно схеме:

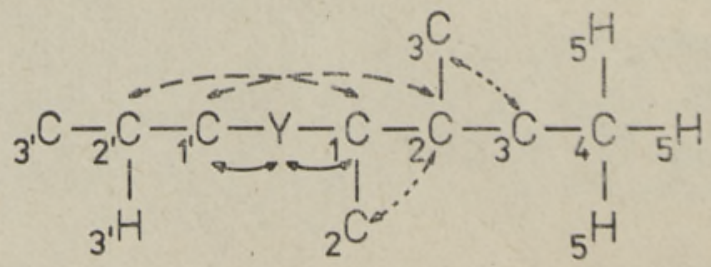

где $i_{1}, i_{2} \ldots$ и $i_{1}^{\prime}, i_{2}^{\prime} \ldots$ - число углеродных атомов в алкильных заместителях $R$ и $R^{\prime}$ в разных положениях относительно центра ионизации $Y$; $h_{1}, h_{2}, \ldots$ и $h_{1}^{\prime}, h_{2}^{\prime} \ldots-$ соответственно число водородных атомов.

В случае алкинов группой $Y$ является $-\mathrm{C} \equiv \mathrm{C}-$. 
Первые адиабатические Пи алкинов $R \mathrm{C} \equiv \mathrm{C}^{\prime}$, эВ

1. $0-0,11,403 ; 11,40 ; 11,40$;

11,$40 ;(11,41) ;(11,406) ;(11,396)$;

$(11,394) ;(11,398) ; c: 11,408$

2. $0-1,10,364 ; 10,37 ; 10,37$; 10,$37 ; 10,38 ;(10,36) ;(10,37)$; c: 10,37

3. $0-2,10,178 ;(10,18)$; c: 10,180

4. $0-3,10,098 ;(10,09)$

5. $0-4,10,067 ;(10,09)$

6. $0-5,10,044 ;(10,03)$

7. $0-6,10,00 ;(9,95)$

8. $0-7,(9,93)$

9. $0-8,(9,91)$

10. $0-9,(9,90)$

11. $0-10,(9,90)$

12. $0-11,(9,90)$

13. $0-12,(9,89)$

14. $1-1,9,562 ; 9,59 ; 9,61$

15. $1-2,9,439$

16. $1-3,9,366 ;(9,37)$

17. $1-4,9,326 ;(9,34)$

18. $1-5,9,302 ;(9,31) ;(9,31)$

19. $1-6,9,289 ;(9,32)$

20. $1-7,(9,30)$

21. $1-8,(9,28)$

22. $1-9,(9,29)$

23. $1-10,(9,28)$

24. $1-11,(9,26)$

25. $2-2,9,323$; $(9,34)$

26. $2-3,9,260 ;(9,28)$

27. $2-4,9,222 ;(9,23) ;(9,22)$

28. $2-5,9,202 ;(9,20)$

29. $2-6,9,190 ;(9,19)$

30. $2-7,(9,17)$
31. $2-8,(9,17)$

32. $2-9,(9,14)$

33. $2-10,(9,17)$

34. $3-3,9,196 ;(9,20),(9,20)$

35. $3-4,(9,17)$

36. $3-5,(9,17)$

37. $3-6,(9,13)$

38. $3-7,(9,14)$

39. $3-8,(9,07)$

40. $3-9,(9,11)$

41. 4-4, 9,125, $(9,14)$

42. $4-5,(9,11)$

43. $4-6,(9,09)$

44. 4-7, $(9,09)$

45. $4-8,(9,10)$

46. $5-5,9,090 ;(9,06)$

47. $5-6,(9,05)$

48. $5-7,(9,09)$

49. $6-6,9,067,(9,03)$

50. $0-i 3,10,049$

51. $0-i 4,10,055$

52. $0-s 4, \mathbf{9 , 9 7 5}$

53. $0-t 4,9,923$

54. $0-i 5, \mathbf{1 0 , 0 1 5}$

55. $1-i 3,9,346$

56. $1-i 4,9,320$

57. $1-t 4,9,276$

58. $1-\mathrm{CH}_{2} \mathrm{C}\left(\mathrm{CH}_{3}\right)_{3}, \mathbf{9 , 2 8 4}$

59. $1-\mathrm{C}\left(\mathrm{CH}_{3}\right)_{2} \mathrm{C}_{2} \mathrm{H}_{5}, \mathbf{9 , 2 1 3}$

60. $1-\mathrm{C}\left(\mathrm{CH}_{3}\right)_{2} \mathrm{C}_{3} \mathrm{H}_{7}, \mathbf{9 , 1 8 3}$

61. $1-\mathrm{C}\left(\mathrm{CH}_{3}\right)_{2} i \mathrm{C}_{3} \mathrm{H}_{7}, \mathbf{9 , 1 5 4}$

62. $2-t 4,9,180$

63. $i 3-i 3,9,171$

64. $t 4-t 4,9,054$

При мечание. В таблице после шифрованной формулы приводятся величины ПИ, полученные методом ФЭС, в скобках - по методу фотоионизации (ФИ). Данные спектроскопического метода обозначают буквой с: Заместители обозначают следующим образом: $\mathrm{H}-0, \mathrm{CH}_{3}-1, \mathrm{C}_{2} \mathrm{H}_{5}-2, n-\mathrm{C}_{3} \mathrm{H}_{7}-3, i-\mathrm{C}_{3} \mathrm{H}_{7}-i 3$ и т. д. Например, $1-t 4$ означает $\mathrm{CH}_{3}-\mathrm{C} \equiv \mathrm{C}-\mathrm{C}\left(\mathrm{CH}_{3}\right)_{3}$. Значения ПИ № 4-6, 16-18, 25, 27, 34, полученные методом ФИ, взяты из [ $\left.{ }^{9}\right]$ и № $7-13,18-24,27-49$ из [10]. Значения ПИ № 1-3, 14 взяты из [4, 11] (кроме выделенных жирным шрифтом).

Для описания изменения ПИ, вызванного алкильными группами $R$ и $R^{\prime}$, используем три типа формальных взаимодействий. тип)

1. Взаимодействие атомов углерода с центром ионизации (C- $\mathrm{Y}$.

2. Взаимодействие между атомами разных заместителей (C- $\mathrm{C}^{\prime}$ тип).

3. Взаимодействие углеродных атомов внутри заместителя (C-Cтип) в разветвленных группах.

Надо найти оптимальную зависимость от числа взаимодействий. Для этого проведен регрессионный анализ исходных данных методом наименьших квадратов в рамках общего уравнения (1)

$$
\Pi И=a_{0}+a_{1} x_{1}+a_{2} x_{2}+\ldots+a_{14} x_{14},
$$

где $x_{1}, x_{2}, \ldots, x_{14}$ показывают число взаимодействий; они высчитываются по структурной формуле. Ниже схематически поясняется какие взаимодействия учитываются каждым регрессором. Дается также их выражение через число углеродных атомов, 


$\begin{array}{llll}x_{1} & { }_{1} \mathrm{C} \longleftrightarrow Y & { }^{\prime} \mathrm{C} \longleftrightarrow Y & x_{1}=i_{1}+i^{\prime}{ }_{1} \\ x_{2} & { }_{2} \mathrm{C} \longleftrightarrow Y & { }^{\prime} \mathrm{C} \longleftrightarrow Y & x_{2}=i_{2}+i^{\prime}{ }_{2}\end{array}$

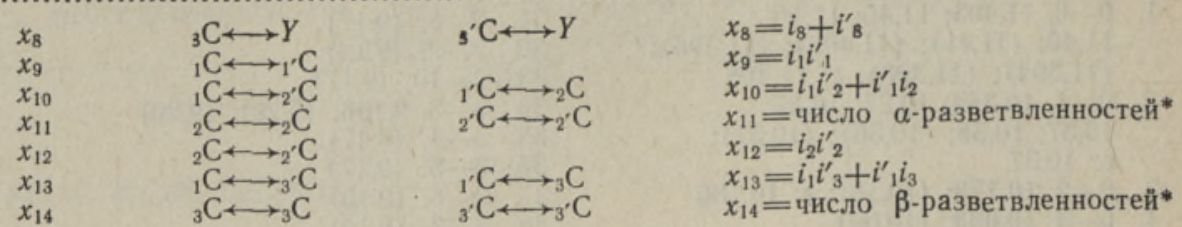

* Примеры: Для $\mathrm{H} Y \mathrm{CH}\left(\mathrm{CH}_{3}\right)_{2} x_{11}=1$, для $\mathrm{H} Y \mathrm{C}\left(\mathrm{CH}_{3}\right)_{3} x_{11}=3$, для $\left(\mathrm{CH}_{3}\right)_{2} \mathrm{CHCH}_{2} Y \mathrm{CH}_{2} \mathrm{C}\left(\mathrm{CH}_{3}\right)_{3} x_{11}=0$ и $x_{14}=4$.

В качестве примера приведен комплект из 14 регрессоров для соединений $\left(\mathrm{CH}_{3}\right)_{3} \mathrm{CC} \equiv \mathrm{CC}\left(\mathrm{CH}_{3}\right)_{3} 2,6,0,0,0,0,0,0,1,6,6,9,0,0$.

После первоначального регрессионного анализа полного комплекта данных 7 значений ПИ 1-алкинов (в табл. 1 номера $7-13$ ), показывающих завышенные результаты, исключены, и регрессорам задана последовательность уменьшения вклада $a_{k} x_{k}$ при росте $k$. В дальнейшем проведена обработка двух наборов данных: первый набор состоит из 97 значений, второй - из 36 особо точных результатов работы [ $\left.{ }^{8}\right]$.

Для выяснения значимости регрессоров уравнения (1) расчетный цикл повторялся исключением каждый раз последнего регрессора уравнения до тех пор, пока последним не оставался $x_{8}$, т. е. учитывались только $\mathrm{C}-Y$-взаимодействия. Из табл. 2 видно, что с учетом только числа С-Y-взаимодействий (регрессия А) оценка ПИ получается грубой. Добавлением регрессоров $\mathrm{C}-\mathrm{C}$-взаимодействия резко улучшаются результаты (регрессии Б, В, Г). Регрессией Г ПИ в обоих наборах описываются практически адекватно точности эксперимента. Благодаря регрессорам $x_{12}, x_{13}$ и $x_{14}$ стандартная ошибка $s$ уменьшается в дальнейшем только на 0,003 эВ.

Таблица 2

Зависимость стандартной ошибки $s$ от числа регрессоров в уравнении (1)

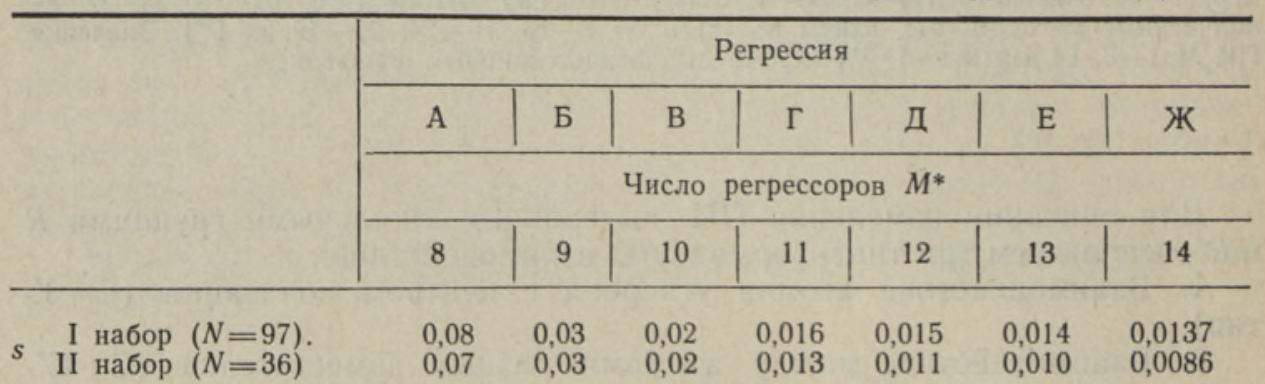

* Число действительных регрессоров при II наборе на два меньше, так как всегда $x_{7}=0$ и $x_{8}=0 . N-$ число значеннй ПИ, включенных в корреляцию.

Оптимальным считается одиннадцатипараметровая регрессия Г. Из табл. 3 видно, что регрессии Г-I и Г-II отличаются хорошими характеристиками $s$ и $R$. Наибольшее отклонение от линии регрессии в I наборе 0,044 эВ и во II наборе только 0,03 эВ.

Между коэффициентами регрессии двух наборов наблюдается некоторое различие. Для сравнения приведены регрессии В-II и Ж-II, причем последняя, наверное, перепараметризована. 
Результаты регрессионного анализа первых Пи по уравнению (1)

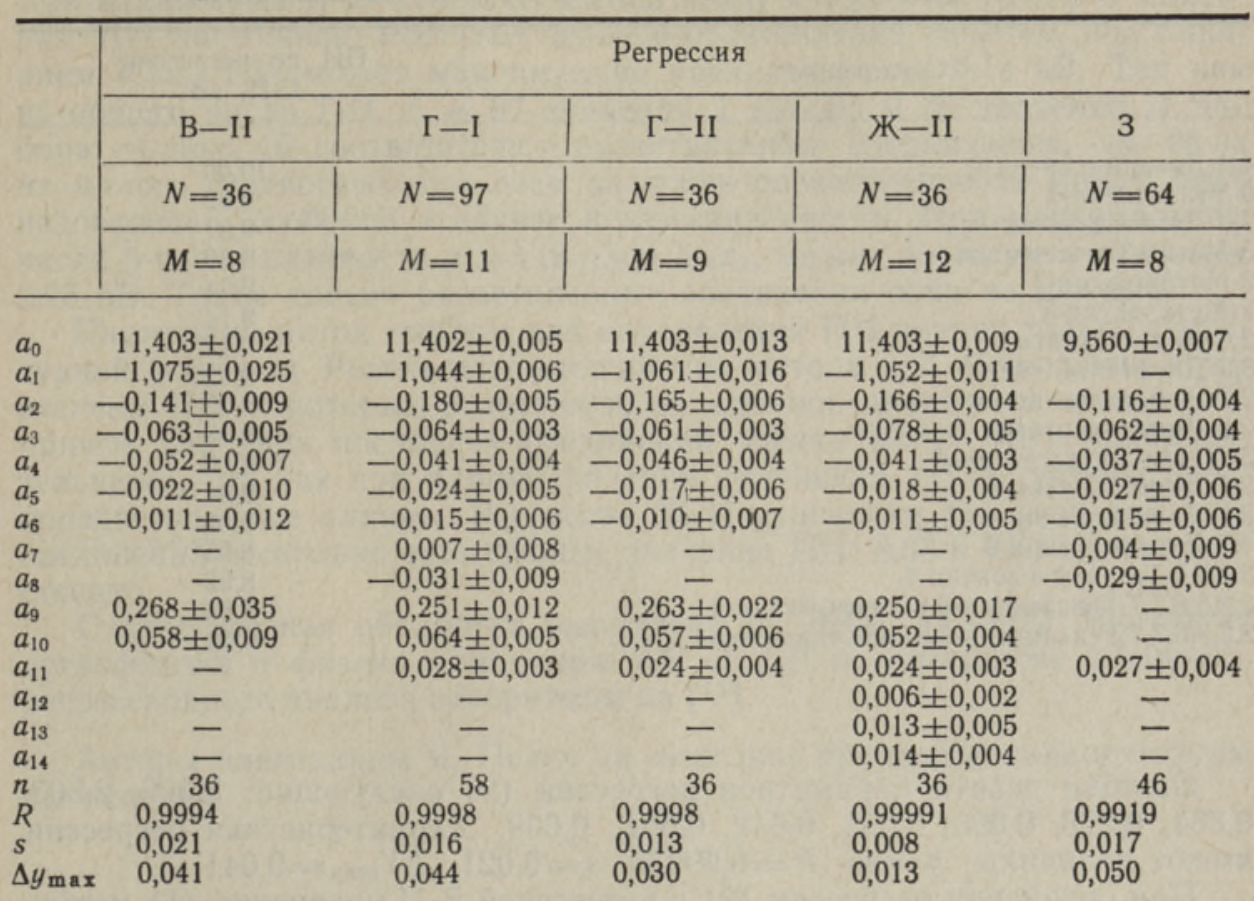

Пр и мечани е: $N-$ число значений ПИ, включенных в корреляцию, $M-$ число регрессоров, $n$ - число соединений, $R$ - множественный коэффициент корреляции, $s$ - стандартная ошибка расчета, $\Delta y_{\max }$ - максимальное отклонение.

Для группы 1 -алкинов ( $\mathrm{HC} \equiv \mathrm{C} R$, где $\left.R=\mathrm{C}_{6} \mathrm{H}_{13}, \ldots, \mathrm{C}_{12} \mathrm{H}_{25}\right)$ все регрессии уравнения (1) дают завышенное значение ПИ (различие порядка 0,1 эВ). В таких соединениях с длинными алкильными цепями, возможно, возникает дополнительное $\mathrm{C}-Y$-взаимодействие, которое не учтено в уравнении (1), или результаты работы [ $\left[{ }^{10}\right]$ в случае 1 -алкинов занижены.

Все соединения с двумя алкильными группами (в табл. 1 № 14-49, 55-64) представляют собой особый выбор, их ПИ можно с хорошей точностью $(s=0,031$ эВ) представить в виде аддитивной зависимости от числа углеродных атомов, однако, если учитывать влияние $\alpha$-разветвления, то стандартная ошибка существенно понижается ( $s=0,017$ эВ). Қак видно из табл. 3 , ПИ этих соединений можно описать равнозначно регрессии 3 или общей регрессии Г-I.

Из $\left[{ }^{1,2}\right]$ в случае многопараметровой корреляции ПИ известна необходимость учета влияния числа водородных атомов в двух соседних позициях по отношению к центру ионизации. Так как число углеродных и водородных атомов функционально связано, то имеется возможность представить аналогичную уравнению (1) зависимость от числа водородных атомов $h_{k}$

$$
\begin{aligned}
\Pi и & =-3,820+7,302\left(h_{1}+h_{1}^{\prime}\right)+2,223\left(h_{2}+h_{2}^{\prime}\right)+0,713\left(h_{3}+h^{\prime}{ }_{3}\right)+ \\
& +0,217\left(h_{4}+h^{\prime}{ }_{4}\right)+0,055\left(h_{5}+h^{\prime}{ }_{5}\right)+0,011\left(h_{6}+h_{6}^{\prime}\right)+0,618 h_{1} h_{1}^{\prime}+ \\
& +0,583\left(h_{1} h^{\prime}{ }_{2}+h^{\prime}{ }_{1} h_{2}\right) .
\end{aligned}
$$


ПИ некоторых известных алкинов $\left[{ }^{12}\right]$ с разветвленной цепью

4,4-Диметилпентин-1

3-Этилпентин-1

3,3-Диметнлгексин-1

3-Метил-3-этилпентин-1

2-Метилгексин-3

2 -Метилоктин-3

2,6 -Диметилгептин-3

3,3-Диметилгептин-4

2,7 -Диметилоктин-4

2,2 -Диметилоктин-3

3-Метил-3-этилгептин-4

10,00

3,6 -Диметилоктин-4

3,3 -Диметилдецин- 4

4,7-Диметилдецин-5

$3,3,6,6$-Тетраметилоктин- 4

9,92

9,81

9,80

9,24

9,14

9,12

9,12

9,09

9,08

9,06

9,04

9,00

8,95

8,92

$2,2,3,6,7,7$-Гексаметил-3,6-диэтилоктин-4

8,55

$2,2,3,6,7,7$-Гексаметил-3,6-дибутилоктин-4

8,43

Ошибки девяти параметров регрессии (2) следующие: 5,006, 2,503, $0,834,0,278,0,093,0,031,0,012,0,041,0,009$. Характеристики регрессии имеют величины: $n=36, R=0,99935, s=0,021, \Delta Y_{\max }=0,041$.

При сравнении регрессии (2) с регрессией В-II уравнения (1) можно легко убедиться, что они находятся в полном функциональном соответствии. Из этого вытекает равноценность описания ПИ с помощью $i_{k}$ или $h_{k}$. Однако описание уравнения (2) «более формальное». Свободный член уравнения (2) имеет нереальную отрицательную величину, и из-за линейной корреляции шкал регрессоров наблюдается большая неопределенность параметров. Поэтому подробнее рассматривается только зависимость ПИ от числа углеродных атомов.

В уравнении (1) свободный член $a_{0}$ соответствует ПИ ацетилена, параметр $a_{1}$ определяет уменьшение ПИ ацетилена в результате введения метильной группы, $a_{2}$ показывает влияние удлинения алкильной цепи в результате добавления второй метильной группы и так до введения восьмой метильной группы. Дальнейшее удлинение алкильной цепи заметно не влияет на ПИ. Если считать величины ПИ первых шести 1 -алкинов из $\left[{ }^{8}\right]$ точными, то коэффициенты $a_{0}-a_{5}$ должны стремиться к величинам: $11,403,-1,039,-0,186,-0,080,-0,031,-0,023$. Взаимодействия по цепи между углеродными атомами ${ }_{1} \mathrm{C} \leftrightarrow{ }_{2} \mathrm{C},{ }_{1} \mathrm{C} \leftrightarrow{ }_{3} \mathrm{C}$ и т. д. не рассматриваются, так как они учтены в величинах $a_{2}, a_{3}, \ldots$ $\ldots, a_{8}$. По представлениям $\left[{ }^{4}\right]$ отрицательность коэффициентов $a_{1}-a_{8}$ показывает, что $\mathrm{C}-Y$-влияние стабилизирует радикал-катион. $\mathrm{B}$ их величинах наблюдается эффект затухания: $\left|a_{1}\right|>\left|a_{2}\right|>\ldots$ $\ldots>\left|a_{6}\right|>\left|a_{7}\right|$. Однако существует отклонение от правила: $\left|a_{7}\right|<$ $<\left|a_{8}\right|$, причем не ясно является это реальным или отражением систематически заниженных оценок адиабатических ПИ у алкилов с длинными цепями.

Взаимное влияние между заместителями и в разветвленных группах между отдельными цепями не позволяет описать ПИ чисто аддитивной схемой как зависимость от числа углеродных атомов. Взаимодействие между первыми углеродами обоих заместителей обусловливает завышение ПИ на 0,26 эВ. Взаимное влияние первого и второго атома углерода также значительное, причем максимально возможное увеличение ПИ из-за члена $a_{10} x_{10}$ уравнения (1) 0,34 эВ. Увеличение расстояния между 
углеродными атомами уменьшает влияние атомов, так как $a_{9}>a_{10}>a_{13}$. Как видно из табл. 2 , влияние членов $a_{12} x_{12}$ и $a_{13} x_{13}$ на точность описания ПИ ничтожное. Рост ПИ из-за $\alpha$-разветвления заметен, под влиянием $a_{11} x_{11}$ ПИ может максимально увеличиться на 0,14 эВ. Так как из общего числа ПИ, т. е. 97 значений I набора и 36 значений II набора, только 15 соответствуют разветвленным соединениям, то из-за их малого статистического веса значение разветвленности может быть недооценено, особенно в случае $\beta$-разветвленности. При максимальном числе $\beta$-разветвленности $x_{14}=18$ член $a_{14} x_{14}$ может достигнуть величины 0,25 эВ. Такие сильно разветвленные соединения пока не изучены.

Настоящий метод требует для определения ПИ знания только структурной формулы. Результаты расширения метода для вычисления неизвестных ПИ некоторых разветвленных алкинов приведены в табл. 4, причем для двух последних соединений оценки могут быть приблизительными, так как при параметризашии уравнения (1) не учтены сильноразветвленные алкины. Регрессия Ж-ІІ уравнения (1) дает для этих соединений несколько завышенные значения ПИ: 8,63 и 8,51 эВ соответственно.

Статистическая обработка выполнена на ЭВМ ЕС-1022 Института астрофизики и физики атмосферы АН ЭССР по программе линейного регрессионного анализа алгоритмом из $\left[{ }^{13}\right]$.

Авторы благодарны Я. Пелту за любезное предоставление программ вычисления.

\section{Л И ТЕРА Т УРА}

1. Пылдоя П. К., Пальм В. А. Реакц. способн. орг. соедин., 4, № 4(14), 786-813 (1967).

2. Коппель И. А., Мельдер У. Х., Пиквер Р. И. Реакц. способн. орг. соеднн., 18, № 3 (67), 366-381 (1981).

3. Price, W. C., Bralsford, R., Harris, P. V., Ridley, R. G. Spectrochim. Acta, 14, № 1, 45-55 (1959).

4. Вилесов Ф. И. В кн.: У $1969,5-45$.

5. Пальм В. А. Основы количественной теории органических реакций. Л., «Химия», 1977.

6. Пылдоя П. Изв. АН ЭССР. Хим. Геол., 25, № 3, 199-205 (1976).

7. Пылдоя П. Изв. АН ЭССР. Физ. матем., 25, № 1, 50-58 (1976).

8. Carlier, P., Dubois, J. E., Masclet, P. G., Mouvier, G. J. Electron Spectrosc. and Relat. Phenom., 7, № 1, 55-67 (1975).

9. Виллем Я. Я., Пиквер Р. И., Сакс О. В. В кн.: III Всесоюзн. конф. по спектроск. вакуумного ультрафиолета и взаимодействию излучения с веществом. Тез. докл. Харьков, Изд. ФТИНТ АН УССР, 1972, 65-66.

10. Ране С., Млртинсон Э. Изв. АН ЭССР. Химия, 27, № 1, 44-45 (1978).

11. Levin, $R$. D., Lias, $S$. G. Ionization potential and appearance potential measurements, 1971-1981. Nat1. Stand. Ref. Date Ser. 71. Washington, 1982.

12. Яснопольский В. Д. Физнко-химические константы органических соединений с ацетиленовой связью. Баку, Изд. АН Азерб. ССР, 1966.

13. Gentleman, W. M. Appl. Statist., 23, № 3, 448-454 (1974).

$\begin{array}{cc}\text { Ннститут физики } & \text { Поступила в редакцию } \\ \text { Академии наук Эстонской ССР } & 18 / \text { III } 1985\end{array}$

Ннститут астрофизики и физики атмосферы Академии наук Эстонской ССР 


\section{IONISATSIOONIPOTENTSIAALI ARVUTAMINE STRUKTUURIOHIKUTEVAHELISTE INTERAKTSIOONIDE ARVU JÄRGI}

\section{Alküünid}

Alküülühendite esimese ionisatsioonipotentsiaali arvutamiseks on esitatud uus meetod, mis arvestab formaalsete molekulisiseste interaktsioonide arvu. Meetod kirjeldab kõiki teadaolevaid alküünide adiabaatseid ionisatsioonipotentsiaale sōltuvusena süsinike arvust alküülgruppides eksperimentaalsele adekvaatse täpsusega. Seitsmeteistkümne hargneva ahelaga alküüni jaoks on ennustatud ionisatsioonipotentsiaali väärtused.

\section{P. POLDOJA, T. VIIK}

\section{CALCULATION OF IONIZATION POTENTIAL BY THE NUMBER OF INTERACTIONS BETWEEN STRUCTURE UNITS}

\section{Alkynes}

A new method for calculating the first ionization potential of alkyl compounds is proposed, which takes into account the number of formal intramolecular interactions. The method describes with experimental accuracy all known adiabatic ionization potentials of alkyls as a function of the number of carbon atoms in alkyl groups. The values of ionization potentials are predicted for seventeen branching alkynes. 